\title{
The Accuracy of Neutrophil/Lymphocyte Ratio in Prediction of Preeclampsia in Low Risk Population
}

\author{
Original Mohamed Samir Eid Sweed ${ }^{1}$, Ahmed Khairy Maqlad ${ }^{2}$, Shaza Mohamed Ahmed \\ Article Elreweny, Osama Ismail Kamel ${ }^{4}$ \\ 1,2,4Department of Obstetrics and Gynecology, Faculty of Medicine, Ain-Shams Univeristy, \\ ${ }^{3}$ Department of Obstetrics and Gynecology, Faculty of Medicine, Alexandria Univeristy, Egypt
}

\begin{abstract}
Background: Hypertensive disorders are the most common medical problems encountered in pregnancy.

Aim: This study aimed to assess the accuracy of neutrophil/lymphocyte ratio (NLR) in prediction of preeclampsia (PET) in low risk population.

Materials and Methods: A prospective comparative cross-sectional study was designed to test the accuracy of neutrophil/ lymphocyte ratio in prediction of preeclampsia in low risk population. The cases were divided into 2 groups; group I included 35 normotensive women at the first 48 hours after delivery with normal blood pressure throughout the pregnancy till full term delivery as a control group and group II included 35 preeclamptic pregnant women at the first 48 hours after delivery with blood pressure greater than or equal to $140 \mathrm{~mm} \mathrm{Hg}$ systolic or greater than or equal to $90 \mathrm{~mm} \mathrm{Hg}$ diastolic on two occasions at least 4 hours apart after 20 weeks of gestation in a woman with a previously normal blood pressure. A complete blood count was taken in three different occasions; the first one was taken just before labour then N/L ratio was calculated (Neutrophil/Lymphocyte ratio. The second one was taken in the first 48 hours after delivery then N/L ratio was calculated (Neutrophil/Lymphocyte ratio. Morover, the third one was taken at early pregnancy before $20^{\text {th }}$ week of gestation from their files then N/L ratio was calculated (Neutrophil/Lymphocyte ratio.

Conclusion: Neutrophil/Lymphocyte ratio cannot predict preeclampsia in low risk population.
\end{abstract}

Key Words: Lymphocytes, neutrophils, neutrophil/lymphocyte ratio, preeclampsia

Received: 19 March 2020, Accepted: 19 June 2021

Corresponding Author: Shaza Mohamed Ahmed Elreweny, Department of Obstetrics and Gynecology, Faculty of Medicine, Alexandria University, Tel.: 01069491030,E-mail: shazaelreweny66@gmail.com

ISSN: 2090-7265, August 2021, Vol.11, No. 3

\section{INTRODUCTION}

Hypertensive disorders are the most common medical problem encountered in pregnancy, affecting up to $15 \%$ of pregnancies and accounting for approximately $25 \%$ of antenatal admissions. They are classified into four categories: Preeclampsia-Eclampsia, chronic hypertension, chronic hypertension with superimposed preeclampsia and gestational hypertension ${ }^{[1,2]}$. Notably preeclampsia (PET) is a major cause of maternal and fetal or neonatal mortality and morbidity ${ }^{[3]}$. The disorder complicates $5 \%-7 \%$ of all pregnancies $^{[4]}$.

Preeclampsia is defined as a new onset of hypertension associated with proteinuria and fluid retention detected for the first time after the $20^{\text {th }}$ week of gestation ${ }^{[5]}$.

Clinical symptoms are hypertension $\geq 140 / 90 \mathrm{~mm}$ $\mathrm{Hg}$ and proteinuria $\geq 0.30 \mathrm{~g} / \mathrm{d}$. Severe cases are often attended by intrauterine growth restriction (IUGR), acute renal failure, HELLP syndrome (Hemolysis, Elevated
Liver enzymes and Low Platelets) or other systemic disturbances ${ }^{[6]}$. It is associated with high risks of preterm delivery, intrauterine growth restriction, placental abruption, renal failure, sub-capsular hepatic hematoma and perinatal mortality, along with maternal morbidity and mortality ${ }^{[2,7]}$. Regarding the pathophysiology of PET, there are many theories highlighting its multifactorial basis. It is probably related to both placental and maternal factors including abnormal placentation, systemic endothelial dysfunction or cell activation, and an angiogenic imbalance favoring anti-angiogenic factors ${ }^{[9]}$.

Preeclampsia is associated with a more extreme maternal systemic inflammatory response than occurs in normal pregnancy. The features of PET arise from the sum of the circulatory disturbances caused by systemic maternal endothelial cell dysfunction or activation. Activated leukocytes will activate endothelium and vice versa ${ }^{[10,11]}$.

Activated leukocytes also release a variety of substances such as cytokine interleukin-8 (IL-8) and 
tumor necrosis factor- $\alpha$ (TNF- $\alpha$ ), which are capable of mediating endothelial function. Interactions between activated leukocytes, platelets, and vascular endothelium are believed to contribute to the vascular injury in this pregnancy disorder. Furthermore; neutrophil activation is believed to be a major component of exaggerated inflammatory responses in the maternal vascular system during $\mathrm{PET}^{[12,13]}$.

Neutrophil lymphocyte ratio (NLR) is a reliable biomarker of low grade inflammation in various clinical conditions, which denotes the ratio of neutrophils, representing the active non-specific inflammatory mediator initiating the first line of defense, to lymphocytes representing the regulatory or protective component of inflammation. It provides prognostic as well as diagnostic information about subclinical inflammation beyond conventional risk factors ${ }^{[14]}$

\section{AIM OF THE WORK}

To assess the the accuracy of neutrophil/lymphocyte ratio (NLR) in prediction of preeclampsia (PET) in low risk population.

\section{PATIENTS AND METHODS}

This is a prospective comparative cross-sectional study. This study was conducted between February 2019 to September 2019. The study was conducted at Obstetrics and Gynecology Department/Maternity Hospital AinShams University. The study was conducted on 70 pregnant women with gestational age $\geq 20$ weeks. They were selected from Ain-Shams Maternity University Hospital after taking an informed written consent to participate in this work. The 70 participants were divided into two groups; Group I included 35 normotensive women at the first 48 hours after delivery with normal blood pressure throughout the pregnancy till full term delivery as a control group and group II included 35 preeclamptic pregnant women at the first 48 hours after labour with blood pressure greater than or equal to $140 \mathrm{~mm} \mathrm{Hg}$ systolic or greater than or equal to $90 \mathrm{~mm} \mathrm{Hg}$ diastolic on two occasions at least 4 hours apart after 20 weeks of gestation in a woman with a previously normal blood pressure. A complete blood count was taken in three different occasions: The first one was taken just before labour then N/L ratio was calculated (Neutrophil/Lymphocyte ratio. The second one was taken in the first 48 hours after delivery then N/L ratio was calculated (Neutrophil/Lymphocyte ratio and the third one was taken at early pregnancy before $20^{\text {th }}$ week of gestation from their files then $\mathrm{N} / \mathrm{L}$ ratio was calculated (Neutrophil/ Lymphocyte ratio.

Preeclamptic patients were diagnosed according to the strict criteria recommended by The American College of Obstetricians and Gynecologists
(ACOG, 2013) $)^{[2]}$. The included women aged from 25 to 35 years. The exclusion criteria were gestational age $<20$ weeks, multiple gestation, chronic hypertension, diabetes mellitus, Preexisting renal or hepatic dysfunction, premature rupture of membranes, ICSI (Intracytoplasmic sperm injection), endometriosis., body mass index (BMI) greater than 30 , systemic autoimmune disease as systemic lupus (SLE) and history of infertility.

Blood sampling collection was done through venipuncture from antecubital vein under a complete aseptic technique sample was taken in two vacutainer tubes. Three milliliters of blood will be on a plain red capped vacutainer tube. After clotting at room temperature, the serum will be separated by centrifugation for 10 minutes at $4000 \mathrm{rpm}$ and then used for assessment of routine chemistry tests (RFT and LFTs). Another $2 \mathrm{ml}$ will be emptied into a lavender tube containing (EDTA) for CBC analysis.

\section{STATISTICAL ANALYSIS}

There is at present limited information regarding the relation between the NLR measures and preeclampsia. So, the present study targets an effect size that is clinically relevant. Using PASS 11 program for sample size calculation, group sample size of 35 women in each group achieves $80 \%$ power to detect a difference of 0.7 between the null hypothesis that both group means of $\mathrm{N} / \mathrm{L}$ ratio are 3.1 and the alternative hypothesis that the mean of group with preeclampsia is 3.1 with estimated group standard deviations of 1.0 and 1.0 and with a significance level (alpha) of 0.05 using a two-sided two-sample t-test.

\section{RESULTS}

A total of 70 women were participated in this study. by comparing the Neutrophil/lymphocyte ratio (1 before labour) between cases and controls we found that the mean was $4.01 \pm 1.53$ and the mean in controls was $3.62 \pm 0.33$ and the $p$ value was 0.14 which is statistically non-significant and by comparing the Neutrophil/ lymphocyte ratio ( 2 after labour) between cases and controls, The mean in cases was $4.22 \pm 1.51$ and the mean in controls was $3.66 \pm 0.35$ and the $p$ value was 0.04 which is statistically significant. After doing the ROC curve, the area under the curve was 0.565 , standared error was 0.076 and $95 \%$ confidence interval's lower bound was .416 and upper bound was 0.713 . So the $\mathrm{N} / \mathrm{L}$ ratio cannot discriminate between cases and controls.

After comparing between neutrophil/lymphocyte ratio 3 between cases and controls, the mean was $3.46 \pm 0.71$ and the mean in controls was $3.25 \pm .57$ and the $p$ value was .17 which is statistically non significant and after doing the ROC curve, the area under the curve was 0.628 , standared error was 0.070 and $95 \%$ confidence interval's lower bound was .491 and upper bound was 0.764 . So the $\mathrm{N} / \mathrm{L}$ ratio cannot predict preeclampsia (Figures 1 and 2). 
Area Under the Curve

95\% Confidence Interval

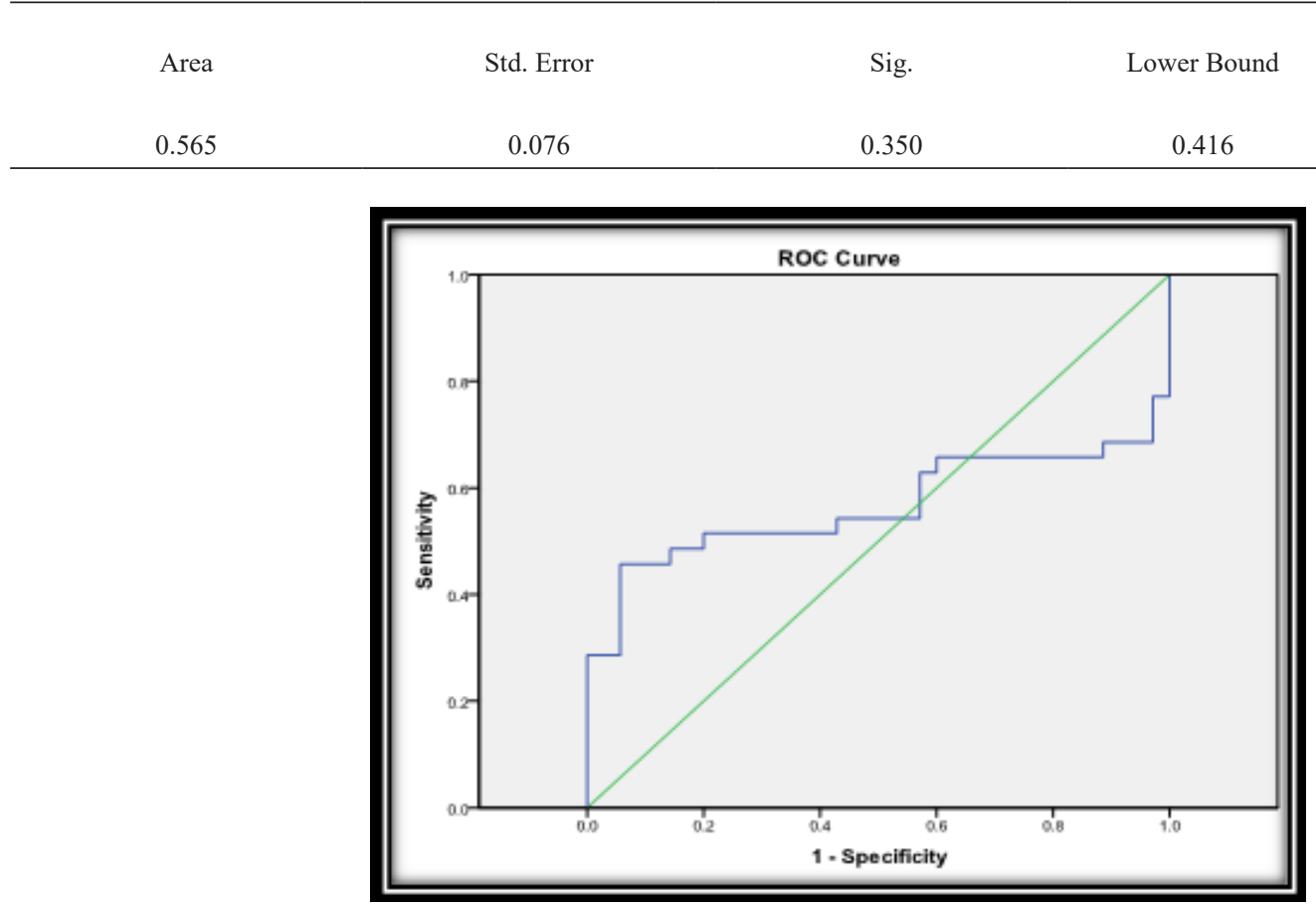

Fig. 1: Diagnostic accuracy of $\mathrm{N} / \mathrm{L}$ ratio before and after labor for diagnosis of preeclampsia

Area Under the Curve

Asymptotic 95\% Confidence Interval

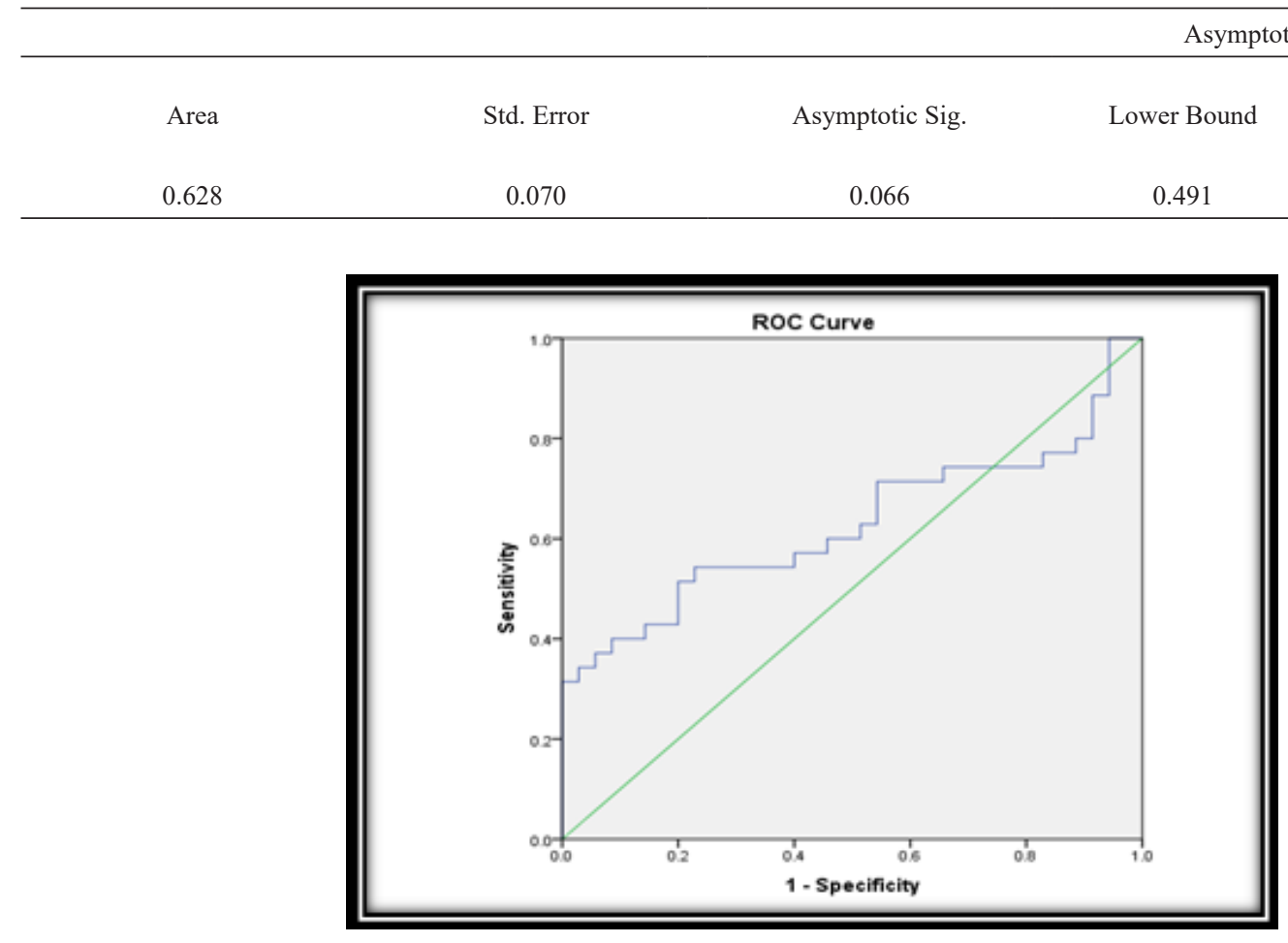

Fig. 2: Predictive ability of N/L ratio for prediction of preeclampsia 
Table 1: Comparison between cases and controls regarding to personal history and clinical examination

\begin{tabular}{|c|c|c|c|c|c|c|c|}
\hline & & \multicolumn{2}{|c|}{ Cases } & \multicolumn{2}{|c|}{ Controls } & \multirow[b]{2}{*}{$t^{*}$} & \multirow[b]{2}{*}{ Pvalue } \\
\hline & & Mean & SD & Mean & SD & & \\
\hline \multicolumn{2}{|c|}{ Age (years) } & 29.11 & 2.90 & 28.83 & 2.56 & 0.44 & $0.66 \mathrm{NS}$ \\
\hline \multirow{2}{*}{\multicolumn{2}{|c|}{ Gestational age (weeks) }} & 36.26 & 1.46 & 37.91 & 1.36 & 4.91 & $<0.001 \mathrm{HS}$ \\
\hline & & $\mathrm{N}$ & $\%$ & $\mathrm{~N}$ & $\%$ & $\mathrm{X} 2 * *$ & Pvalue \\
\hline \multirow[t]{6}{*}{ Gravidity } & 1.00 & 12 & $34.3 \%$ & 12 & $34.3 \%$ & & \multirow{4}{*}{$0.99 \mathrm{NS}$} \\
\hline & 2.00 & 9 & $25.7 \%$ & 9 & $25.7 \%$ & & \\
\hline & & & & & & & \\
\hline & 3.00 & 8 & $22.9 \%$ & 7 & $20.0 \%$ & 0.14 & \\
\hline & $>4.00$ & 6 & $17.1 \%$ & 7 & $20.0 \%$ & & \\
\hline & .00 & 14 & $40.0 \%$ & 12 & $34.3 \%$ & & \multirow{5}{*}{$0.77 \mathrm{NS}$} \\
\hline \multirow[t]{4}{*}{ Parity } & 1.00 & 12 & $34.3 \%$ & 11 & $31.4 \%$ & \multirow{4}{*}{1.23} & \\
\hline & & & & & & & \\
\hline & 2.00 & 6 & $17.1 \%$ & 6 & $17.1 \%$ & & \\
\hline & $>3.00$ & 3 & $8.6 \%$ & 6 & $17.1 \%$ & & \\
\hline \multirow[t]{2}{*}{ Previous } & no & 25 & $71.4 \%$ & 30 & $85.7 \%$ & \multirow{3}{*}{2.12} & 0.15 \\
\hline & & & & & & & \\
\hline abortion & yes & 10 & $28.6 \%$ & 5 & $14.3 \%$ & & NS \\
\hline \multicolumn{2}{|l|}{ Weight (Kg) } & 74.20 & 8.98 & 75.09 & 8.43 & 0.43 & $0.67 \mathrm{NS}$ \\
\hline \multicolumn{2}{|l|}{ Height (cm) } & 162.57 & 5.36 & 163.71 & 6.40 & 0.81 & $0.42 \mathrm{NS}$ \\
\hline \multicolumn{2}{|l|}{$\mathrm{BMI}(\mathrm{Kg} / \mathrm{m} 2)$} & 28.20 & 3.97 & 27.99 & 2.73 & 0.25 & $0.80 \mathrm{NS}$ \\
\hline \multicolumn{2}{|l|}{ SBP } & 142.43 & 12.62 & 109.71 & 11.24 & 11.45 & $<0.001 \mathrm{HS}$ \\
\hline \multicolumn{2}{|l|}{ DBP } & 96.00 & 8.98 & 69.57 & 7.71 & 13.22 & $<0.001 \mathrm{HS}$ \\
\hline
\end{tabular}


Table 2: Comparison between cases and controls regarding to Lab investigations

\begin{tabular}{|c|c|c|c|c|c|c|}
\hline & \multicolumn{2}{|c|}{ Cases } & \multicolumn{2}{|c|}{ Controls } & \multirow[t]{2}{*}{$\mathrm{t}^{*}$} & \multirow[t]{2}{*}{ Pvalue } \\
\hline & Mean & SD & Mean & SD & & \\
\hline proteinurea (mg $\backslash d 124$ hour) & 2745.31 & 619.59 & 132.18 & 15.68 & 24.94 & $<0.001 \mathrm{HS}$ \\
\hline PLT & 239.74 & 80.53 & 247.60 & 91.30 & 0.38 & $0.70 \mathrm{NS}$ \\
\hline $\mathrm{HB}(\mathrm{mg} / \mathrm{dl})$ & 11.01 & 1.24 & 10.68 & 1.32 & 0.94 & $0.35 \mathrm{NS}$ \\
\hline ALT & 40.86 & 33.91 & 16.00 & 11.10 & 4.12 & $<0.001 \mathrm{HS}$ \\
\hline AST & 31.71 & 22.20 & 30.54 & 16.05 & 0.25 & $0.80 \mathrm{NS}$ \\
\hline s.creatinine & 0.70 & 0.21 & 0.59 & 0.17 & 2.24 & $0.03 \mathrm{~S}$ \\
\hline Neutrophils1 & 70.83 & 9.11 & 75.91 & 4.05 & 3.02 & $0.004 \mathrm{HS}$ \\
\hline Lymphocytes 1 & 19.77 & 6.38 & 21.21 & 2.75 & 1.23 & $0.22 \mathrm{NS}$ \\
\hline Neutrophils2 & 72.02 & 7.63 & 75.67 & 4.02 & 2.50 & $0.02 \mathrm{~S}$ \\
\hline Lymphocytes 2 & 18.97 & 5.97 & 20.88 & 2.73 & 1.73 & $0.09 \mathrm{NS}$ \\
\hline \multicolumn{7}{|l|}{ Neutrophil/Lymphocyte } \\
\hline ratio 1 & 4.01 & 1.53 & 3.62 & 0.33 & 1.50 & $0.14 \mathrm{NS}$ \\
\hline \multicolumn{7}{|l|}{ Neutrophil/Lymphocyte } \\
\hline ratio 2 & 4.22 & 1.51 & 3.66 & 0.35 & 2.11 & $0.04 \mathrm{~S}$ \\
\hline Netrophils3 & 71.67 & 4.48 & 74.49 & 2.57 & 3.24 & $0.002 \mathrm{HS}$ \\
\hline Lymphocytes3 & 21.61 & 4.79 & 24.47 & 10.00 & 1.52 & $0.13 \mathrm{NS}$ \\
\hline \multicolumn{7}{|l|}{ Neutrophil/ Lymphocyte } \\
\hline ratio 3 & 3.46 & 0.71 & 3.25 & 0.57 & 1.39 & $0.17 \mathrm{NS}$ \\
\hline
\end{tabular}

*Student $t$ test 


\section{DISCUSSION}

Preeclampsia (PE) is defined as a new onset of hypertension associated with proteinuria and fluid retention detected for the first time after the $20^{\text {th }}$ week of gestation. $\mathrm{PE}$, or "toxemia of pregnancy" []].

Although the causes of PE are completely unknown, one of the responsible mechanisms is thought to be activation of inflammatory systems with predominant involvement of cytokines and chemokines ${ }^{[14]}$. Inflammatory statuses of PE have been evaluated using several biomarkers such as C-reactive protein (CRP) and mean platelet volume (MPV). Recently, neutrophil/ lymphocyte ratio (NLR) and platelet/lymphocyte ratio (PLR), which can be derived from the complete blood count (CBC), have been studied as novel markers of prognosis in patients with $\mathrm{PE}^{[15,16]}$.

These studies showed that NLR could be involved in inflammatory and thrombotic processes. We compared NLR and levels in patients with PE with matched normal pregnant women and evaluated whether there is a predictive value of neutrophil/lymphocyte ratio with preeclampsia or not. This comparative cross sectional study was conducted on 70 women distributed into 2 groups divided equally to pereclamptic cases and non-preeclamptic controls, each group is 35 women in fertile age ranged between 25-35 years and the study was held at the Obstetrics and Gynecology Department/Maternity Hospital, Ain-Shams University between February 2019 and October 2019, complete blood count was taken from all patients in three occasions before $20^{\text {th }}$ week of gestation at time of labour and the first 48 hours after delivery.

By comparing the neutrophil/lymphocyte ratio 1 "before labour" between cases and controls, the mean in cases was $4.01 \pm 1.53$ and the mean in controls was $3.62 \pm 0.33$ and the $p$ value was 0.14 which is statistically non-significant. Also, by comparing the neutrophil/lymphocyte ratio 2 "after labour" between cases and controls, the mean in cases was $4.22 \pm 1.51$ and the mean in controls was $3.66 \pm 0.35$ and the $p$ value was 0.04 which is statistically significant.

After doing the ROC curve, the area under the curve was 0.565 , standared error was 0.076 and $95 \%$ confidence interval's lower bound was 0.416 and upper bound was 0.713 . So, the neutrophil/lymphocyte ratio cannot discriminate between cases and controls.

Also, there was no statistically difference between the two groups regarding neutrophil/lymphocyte ratio before $20^{\text {th }}$ weeks of gestation as the mean was $3.46 \pm 0.71$ and the mean in controls was $3.25 \pm .57$ and the $p$ value was 0.17 which is statistically non significant. After doing the ROC curve, the area under the curve was 0.628 , standared error was 0.070 and $95 \%$ confidence interval's lower bound was 0.491 and upper bound was 0.764 .
There was marked increase in neutrophils level before labour and in the first 48 hours after labour between the two groups ( $p=0.004$ and $p=0.02$ respectively) which is statistically significant but the net neutrophil/lymphocyte ratio doesn't statistically affected. So, the net result in this study showed that neutrophil/lymphocyte ratio cannot predict preeclampsia.

Throughout our study, we found that there was no statistically significant difference between the two groups in terms of median age, gravidity, parity, number of previous abortion, weight, height, body mass index, haemoglobin level, platelets count, lymphocytic count before labour and in the first 48 hours after labour $(p=0.66, p=0.99$, $p=0.77, p=0.15, p=0.67, p=0.42, p=0.80, p=0.70, p=0.35$, $p=0.22$ and $p=0.09$, respectively) (Table 1 ).

In contrast, we found that preeclamptic pregnants had smaller gestation weeks than that of normal pregnant women $(p=<0.001)$ which is statistically highly significant, in addition, systolic blood pressure and diastolic blood preesure were higher in preeclamptic women than normal group ( $p=<0.001$ and $p=<0.001$, respectively) which is statistically highly significant too (Table 2 ).

These results are in agreement with the results of Yavuzcan et al. ${ }^{[17]}$ who conducted a study on 101 women divided into 3 groups (30 patients with preeclampsia, 36 healthy pregnant women and 35 healthy non-pregnant women) reported that neutrophil/lymphocyte ratio was not meaningfully differrent in patients with preeclampsia and healthy pregnant women $(p=0.721)$ with a mean of $4.04 \pm 2.03$ for preeclamptic women and a mean of $3.76 \pm 1.28$ for healthy pregnant women, Also, BurakYücel et al. ${ }^{[18]}$ who conducted their retrospective cohort study on 219 patients (27 had mild PE, 82 had severe PE, and 110 were healthy, normotensive pregnant patients) found that $(p=0.423)$ and area under the curve for NLR is (0.636). So, there was no statistically difference in NLR between mild preeclampsia, severe preeclampsia or healthy pregnant groups.

The study of Toptas et al. ${ }^{[19]}$ which was done on (93 women with preeclampsia and 94 normal pregnant women) matched for both maternal age and gestastional age showed that the mean of neutrophil/lymphocyte ratio was $7.4 \pm 5.2$ for preeclamptic women and was $7.2 \pm 3.7$ for healthy pregnant women $(P=0.7)$ which is statistically non-significant, the mean of platelet/lymphocyte ratio was $134.4 \pm 64.5$ for preeclamptic women and was $130.5 \pm 86.2$ for healthy pregnant women $(P=0.898)$ which is statistically non-significant. Also, the mean of neutrophil/lymphocyte ratio was $5.9 \pm 3.5$ for mild preeclampsia $(\mathrm{n}=27)$ and was $6.8 \pm 3.9$ for severe preeclampsia $(\mathrm{n}=66)(P=0.314)$ which is statistically non-significant, the mean of platelet/lymphocyte ratio was $122.9 \pm 92.0$ for mild preeclampsia $(n=27)$ and 
was $149.8 \pm 67.3$ for severe preeclampsia $(n=66)(P=0.024)$ which is statistically significant so PLR was found to be associated with the severity of PE, whereas NLR was not and both values can't predict preeclampsia. This finding may berelated to cytokine-dependent defective maternal immune activation in PE pathogenesis. In addition, PLR may also be an indirectly available and simple reflector for degree of immune activation in PE. But the data about the importance of PLR and NLR in PE are insufficient and further research is required to elucidate the significance of PRL and NLR in PE.

In contrast to our study, Balta et al. ${ }^{[20]}$ made their pilot study on 203 pregnant women (73 normotensive pregnants, 23 pregnants with mild preeclampsia and 107 pregnants with severe preeclampsia) and were retrospectively analyzed then neutrophil to lymphocyte ratios were compared between the study groups. The study results denoted that neutrophil/lymphocyte ratio in preeclamptic group was significantly higher than that of normal group $(p=0.023)$ and area under ROC curve was found statistically significant $(p=0.023)$.

However, there was no statistically significant relationship between NLR and severity and the findings showed that the measurement of NLR periodically may be useful to predict high-risk pregnancies in terms of preeclampsia, but further studies are needed to determine its contribution. But they agreed with our study regarding that there was no statistically significant difference between two groups in terms of median age, gravida and parity and also preeclamptic pregnants had smaller gestation weeks than that of normal pregnant women $(p=0.005)$ which is statistically significant.

Serin et $a .^{[21]}$ also studied 30 healthy pregnant females, 37 females with mild preeclampsia and 40 with severe preeclampsia. They found that NLR was significantly high in the preeclamptic patients compared to the healthy pregnant patients. NLR was significantly higher in the severe preeclampsia group than in the mild preeclampsia group. So, they concluded that NLR could predict the severity of the disease.

\section{CONCLUSION}

Neutrophil/Lymphocyteratio cannot predict preeclampsia in low risk population.

\section{CONFLICT OF INTEREST}

There are no conflicts of interests.

\section{REFERENCES}

1. Butalia S, Audibert F, Côté AM, Firoz T, Logan AG, Magee LA \& Nerenberg KA (2018): Hypertension Canada's 2018 guidelines for the management of hypertension in pregnancy. Canadian Journal of Cardiology, 34(5), 526-531.

2. American College of Obestetrics and Gynecologists (2013): Task Force on Hypertension in P. Hypertension in pregnancy. Report of the American College of Obstetricians and Gynecologists' Task Force on Hypertension in Pregnancy. Obstet Gynecol; 122(5):1122-31.

3. Sibai B, Dekker G, Kupferminc M (2005): Preeclampsia. Lancet; 365(9461):785-99.

4. Wagner R, Miller S, O'Shaughnessy A (2004): Area wide electronic booking: a revolution in the management of health and well being. Stud Health Technol Inform; 100:169-73.

5. Salam RA, Das JK, Ali A, Bhaumik S \& Lassi ZS (2015): Diagnosis and management of preeclampsia in community settings in low and middle-income countries. Journal of family medicine and primary care, 4(4), 501.

6. Cornelius DC and Wallace K (2019): Decidual natural killer cells: A critical pregnancy mediator altered in preeclampsia. EBioMedicine, 39, 31-32.

7. Mac KAP, Berg CJ, Liu X, Duran C, Hoyert DL (2011): Changes in pregnancy mortality ascertainment: United States, 1999-2005. Obstet Gynecol; 118(1): 104-10.

8. Fisher SJ (2015): Why is placentation abnormal in preeclampsia?. American journal of obstetrics and gynecology, 213(4), S115-S122.

9. Mihu, D., Razvan, C., Malutan, A., \& Mihaela, C. (2015): Evaluation of maternal systemic inflammatory response in preeclampsia. Taiwanese Journal of Obstetrics and Gynecology, 54(2), 160-166.

10. Tannetta D, Masliukaite I, Vatish M, Redman C \& Sargent I (2017): Update of syncytiotrophoblast derived extracellular vesicles in normal pregnancy and preeclampsia. Journal of reproductive immunology, 119, 98-106.

11. Kurtoglu, E., Kokcu, A., Celik, H., Tosun, M., \& Malatyalioglu, E. (2015): May ratio of neutrophil to lymphocyte be useful in predicting the risk of developing preeclampsia? A pilot study. The Journal of Maternal-Fetal \& Neonatal Medicine, 28(1), 97-99 
12. Yıldız, Ç., Karakuş, S., Akkar, Ö. B., Topbaş, T., Çetin, M., Yanık, A., \& Çetin, A. (2016): The Significance of Neutrophil-Lymphocyte Ratio and Mean Corpuscular Volume in Diagnosis of Preeclampsia. Gynecology Obstetrics \& Reproductive Medicine, 22(2), 75-79.

13. LaMarca B, Cornelius DC, Harmon AC, Amaral LM, Cunningham MW, Faulkner JL \& Wallace K (2016): Identifying immune mechanisms mediating the hypertension during preeclampsia. American Journal of Physiology-Regulatory, Integrative and Comparative Physiology, 311(1), R1-R9.

14. Dogan K, Guraslan H, Senturk MB, Helvacioglu C, Idil S, Ekin M (2015): Can Platelet Count and Platelet Indices Predict the Risk and the Prognosis of Preeclampsia? Hypertens Pregnancy; 34(4):434-442.

15. Han L, Liu X, Li H, Zou J, Yang Z, Han J, Huang $\mathrm{W}$, et al. (2014): Blood coagulation parameters and platelet indices: changes in normal and preeclamptic pregnancies and predictive values for preeclampsia. PLoS One. 2014; 9(12):e114488.

16. Yavuzcan A, Caglar M, Ustun Y, Dilbaz S, Yidiz E, Ozbilgec S \& Ozdemir I (2014). Mean platelet volume, neutrophil-lymphocyte ratio and platelet-lymphocyte ratio in severe preeclampsia. Ginekologia polska, 85(3).

17. Yücel B and Ustun B (2017): Neutrophil to lymphocyte ratio, platelet to lymphocyte ratio, mean platelet volume, red cell distribution width and plateletcrit in preeclampsia. Pregnancy Hypertension: An International Journal of Women's Cardiovascular Health, 7, 29-32.

18. Toptas M, Asik H, Kalyoncuoglu M, Can E, Can MM (2016): Are Neutrophil/ Lymphocyte Ratio and Platelet/Lymphocyte Ratio Predictors for Severity of Preeclampsia? J Clin Gynecol Obstet; 5(1):27-31.

19. Balta S, Kurtoglu E, Kucuk U, Demirkol S, Ozturk C (2014): Neutrophil-lymphocyte ratio as an important assessment tool. Expert Rev Cardiovasc Ther; 12(5):537-8.

20. Serin S, Avci F, Ercan O, Kostu B, Bakacak M, Kiran H (2016): Is neutrophil/lymphocyte ratio a useful marker to predict the severity of preeclampsia? Pregnancy Hypertens; 6(1):22-5. 